\title{
ПРИМЕНЕНИЕ АППАРАТА НЕЧЕТКОЙ ЛОГИКИ К РЕШЕНИЮ ЗАДАЧ УПРАВЛЕНИЯ В ТЕЛЕКОММУНИКАЦИОННЫХ КОМПАНИЯХ
}

\section{APPLICATION OF THE APPARATUS OF FUZZY LOGIC TO SOLVING MANAGEMENT PROBLEMS IN TELECOMMUNICATION COMPANIES}

\section{K. Matrokhina}

Summary. The article presents an information traffic control algorithm in telecommunication networks, built using the fuzzy logic apparatus. As the base of fuzzy control algorithm, the Mamdani algorithm was chosen. Corresponding operations of fuzzy inference have been determined, allowing to give the control algorithm "flexibility" based on taking into account the accumulated practical experience of staff in the process of operating a telecommunication network. Initial dataset has been generated based on the technical characteristics of the network, as well as on the basis of expert data obtained during its operation. The required linguistic variables used to control the network were specified, for these variables the necessary membership functions of three types are constructed. The base of fuzzy inference rules is formed, on the basis of which the value of the output linguistic variable is found. The results obtained allowed to identify the most effective model of the membership function, which must be used for adequate fuzzy control.

Keywords: control, information traffic, telecommunications network, bandwidth, fuzzy logic, Mamdani algorithm, membership functions, queuing system.

\section{Введение}

жедневное увеличение числа пользователей телекоммуникационных сетей и объемов передаваемой информации обусловливают повышение требований к пропускной способности каналов связи и к качеству сетевого обслуживания в целом.

Построение механизмов, алгоритмов и протоколов, позволяющих реализовывать требуемое качество обслуживания для различных приложений и услуг сетей связи, постоянно являются предметом исследования отечественных и зарубежных ученых. К проблеме повышения качества обслуживания сети и управления информационным трафиком, в частности, в своих трудах обращались Симаков Д.В. [1], Bogatin E. [2], Bouillard A. [3], Levy B.[4], Moscholios I. [5], Zukerman M. [6] и др.

\author{
Матрохина Кристина Васильевна \\ Аспирант, Санкт-Петербургский горный \\ университет \\ k.matrokhina@mail.ru
}

Аннотация. В статье представлен алгоритм управления информационным трафиком в телекоммуникационных сетях, построенный с применением аппарата нечеткой логики. В качестве основы алгоритма нечеткого управления выбран алгоритм Мамдани. Определены соответствующие операции нечеткого вывода, позволяющие придать алгоритму управления «гибкость», основанную на учете накопленного практического опыта обслуживающего персонала в процессе эксплуатации телекоммуникационной сети. Сформировано множество исходных данных на основе технических характеристик сети, а также на основе экспертных данных, полученных в процессе ее эксплуатации. Заданы требуемые лингвистические переменные, используемые для управления сетью, для этих переменных построены необходимые функции принадлежности трех видов. Сформирована база правил нечеткого вывода, на основе которых находится значение выходной лингвистической переменной. Полученные результаты позволили выявить наиболее эффективную модель функции принадлежности, которую необходимо использовать для адекватного нечеткого управления.

Ключевые слова: управление, информационный трафик, телекоммуникационная сеть, пропускная способность, нечеткая логика, алгоритм Мамдани, функции принадлежности, система массового обслуживания.

В последнее время увеличилось количество работ, в которых для управления трафиком применяется аппарат нечеткой логики. Например, в [7] описан метод маршрутизации для беспроводной сенсорной сети, базирующийся на средствах нечеткого вывода. В [8] предложена методика управления процессом передачи информационного трафика за счет регулирования уровня загрузки буферов сетевых устройств. Методика включает применение адаптивного нечеткого контроллера и нейронные сети.

Возрастающая популярность использования теории нечетких множеств для управления технологическими процессами, связана, в первую очередь, с тем, что не требуется создавать подробных функциональных моделей этих процессов, необходимо только сформулировать правила поведения в форме нечетких ус- 
ловных суждений типа IF ... THEN. Для моделирования нечетких систем существует ряд инструментальных средств, тем не менее, как правило, используется пакет расширения Fuzzy Logic Toolbox вычислительной среды MATLAB.

Для решения задачи управления трафиком в телекоммуникационных сетях применяются различные алгоритмы, наиболее известными из которых являются алгоритмы TailDrop, PI, RED. Данные алгоритмы показали свою работоспособность и приемлемую эффективность, но, тем не менее, и они имеют определенные недостатки. Так, например, алгоритм TailDrop вызывает перегрузку. Алгоритм RED изменяет вероятность сброса по линейному закону, но не позволяет эффективно регулировать нелинейную динамику трафика. Алгоритм PI требует представительной обучающей выборки в виду использования машинного обучения.

Для снижения недостатков упомянутых выше алгоритмов управления трафиком в телекоммуникационных сетях необходимо использовать аппарат нечеткой логики, прикладные аспекты применения которого нашли отражение в работах Паклина Н. [9], Штовба С.Д. [10], Асадулаева Р.Г. [11], Емельянова С. Г.[12], Бауэра П. [13].

Алгоритм нечеткого управления информационным трафиком в телекоммуникационных сетях. Рассмотрим предлагаемую систему нечеткого управления информационным трафиком в телекоммуникационных сетях на основе алгоритма Мамдани, в которой в отличие от известных подобных механизмов в базе правил нечетких продукций реализованы новые сочетания входных лингвистических переменных (контролируемых параметров).

Алгоритм Мамдани описывает несколько последовательно выполняющихся этапов: формирование базы правил $\rightarrow$ фаззификация, $\rightarrow$ агрегирование подусловий$\rightarrow$ активизация подзаключений $\rightarrow$ аккумулирование заключений $\rightarrow$ дефаззификация[7].

Указанный алгоритм примечателен тем, что реализует принцип «черного ящика», при этом каждый последующий этап получает на вход значения, полученные на предыдущем шаге.

В качестве объекта, на котором был апробирован предложенный алгоритм, была выбрана телекоммуникационная сеть транспортно-энергетической компании, офисы которой территориально расположены в разных районах Санкт-Петербурга. Все подразделения компании, при взаимодействии между собой и с контрагентами, используют для передачи информации практически все виды трафика.
Организация пользуется услугами Интернет-провайдера, который, в соответствии с договором, предоставляет скорость обмена информацией 100 Мбит/с. Отдел информатизации, в составе которого работают высококвалифицированные специалисты, обеспечивает функционирование сети телекоммуникаций в соответствии с установленными внутренними и внешними требованиями.

В существующих условиях наиболее жесткие требования к качеству обмена предъявляют такие виды информации как видео, аудио, данные. Поскольку компания использует трафик, наполненный указанными видами информации, то для корпоративной телекоммуникационной сети используется технология качества обслуживания (QoS). Благодаря данной технологии можно распределять трафик по категориям обслуживания и приоритетам. Для информационного трафика с высоким приоритетом обеспечивается гарантированное качество обслуживания и лучшие условия передачи, поэтому для транзакций и голоса выделяется $60 \%$ доступной полосы пропускания, а для серфинга порядка 5-10\%. Таким образом, используемая технология качества обслуживания позволяет применить новый метод нечеткого управления информационным трафиком, при этом адекватно задавать значения лингвистическим переменным.

\section{Функшионирование алгоритма нечеткого управления}

Рассмотрим функционирование предложенного алгоритма.

1. Формирование множества исходных данных осуществляется на основе заданных и измеряемых технических характеристиках сети, а также на основе экспертных данных, полученных в процессе эксплуатации. Входными данными алгоритма являются значения переменных, характеризующих информационный трафик. В данном случае это приоритет трафика, его задержка и суточная загруженность сети. Каждая переменная характеризуется соответствующим числовым значением. Значение приоритета изменяется от 0 до 100\%, значение задержки - от 0 до 200 мс, значение суточной загруженности сети - интервалы времени суток от 0 до 24 ч. Выходной управляемой переменной является пропускная способность для пользователя, значение которой изменяется от 0 до 100 Мбит/с. На рис. 1 схематично представлена система нечеткого управления (контроллера) пропускной способностью.

2. Для входных и выходной переменных задаются соответствующие термы, которым присваиваются интервалы значений. 


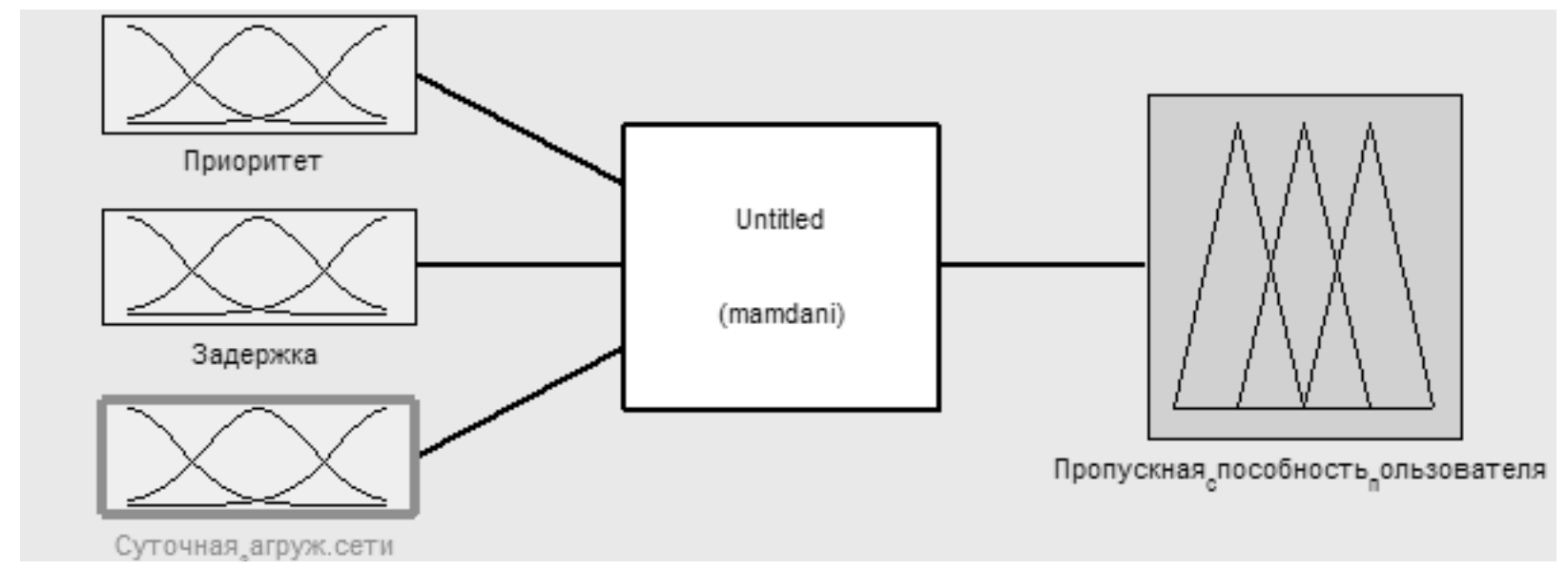

Рис. 1. Система нечеткого управления (контроллера) пропускной способностью
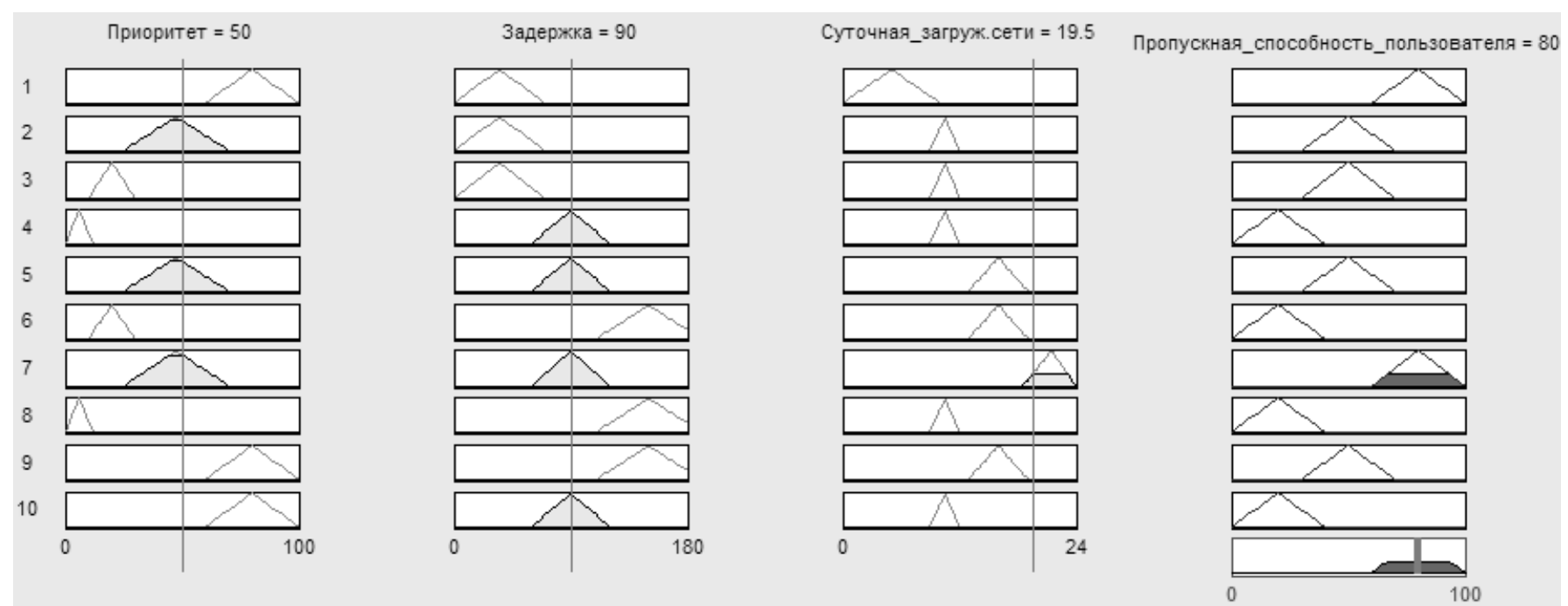

Рис. 2. Визуальное представление правила для лингвистических переменных с треугольной t-нормой

Для переменной «приоритет» трафика заданы следующие лингвистические переменные:

«Стандарт»: 0-10\%;»Экспресс»: 10-30\%; «Премиум»: 25-70\%; «Максимум»:60-100\%.

Для переменной «задержка» заданы следующие термы:

«Маленькая»: 0-79 мс; «Средняя»: 66-135 мс; «Большая»: 120-200 MC.

Для переменной «суточная загруженность сети» трафика заданы следующие термы:

«Маленькая»: 0-10 ч, 12-13 ч, 19-24 ч.; «Средняя»: 9-12 ч; «Большая»: 13-18 ч.
Для выходной переменной «пропускная способность пользователя» заданы следующие термы:

«Маленькая»: 0-40 Мбит/с.; «Средняя»: 30-70 Мбит/с; «Большая»: 60-100 Мбит/с.

Используя указанные лингвистические переменные и их значения можно сформировать соответствующие функции принадлежности. В каждом отдельном случае построения функции принадлежности формулируются свои требования и обоснования к выбору именно такого построения. Одним из основных методов построения указанных функций является метод экспертных оценок. В рассматриваемом случае используются оценки специалистов отдела информатизации, накопленные в процессе эксплуатации сети телекоммуникаций. Типизация функций принадлежности в контексте решаемой задачи 


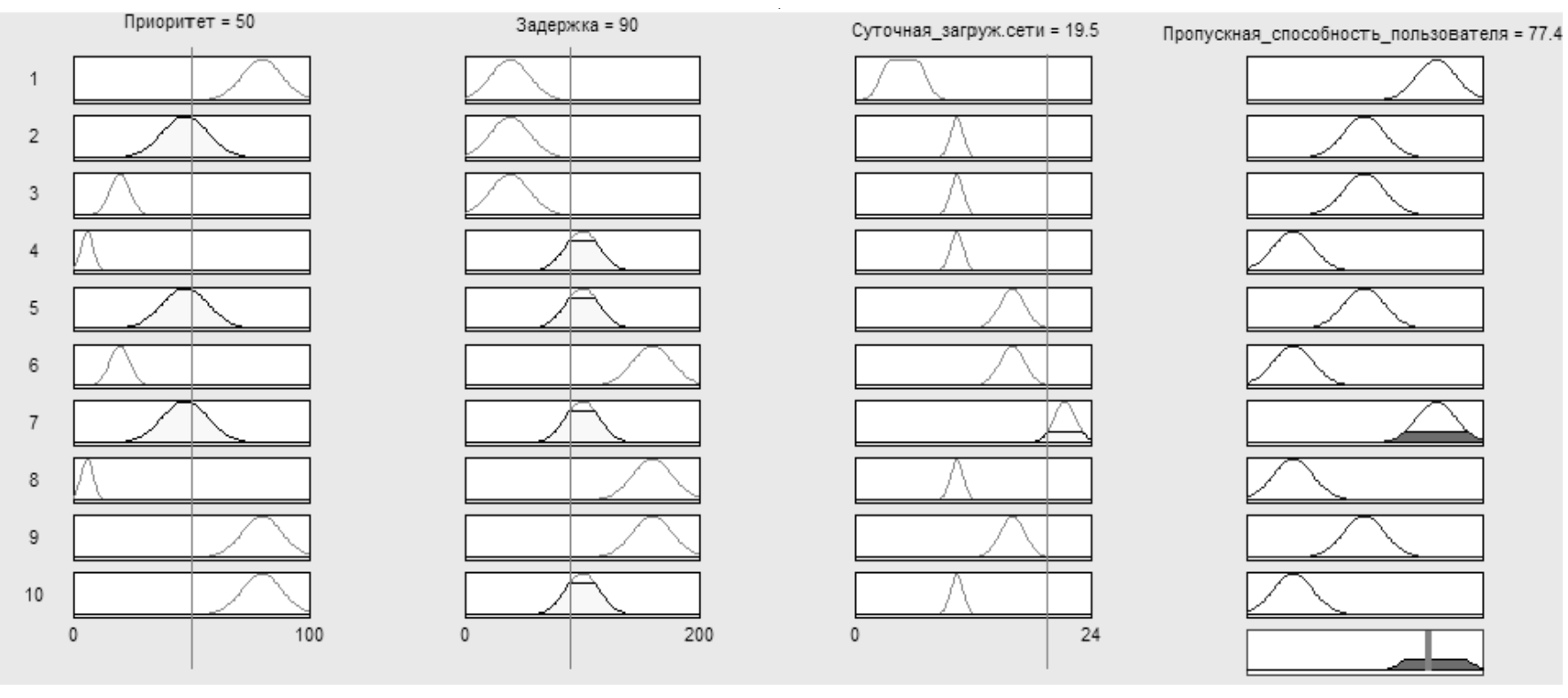

Рис. 3. Визуализация нечеткого вывода с гауссовскими функциями принадлежности

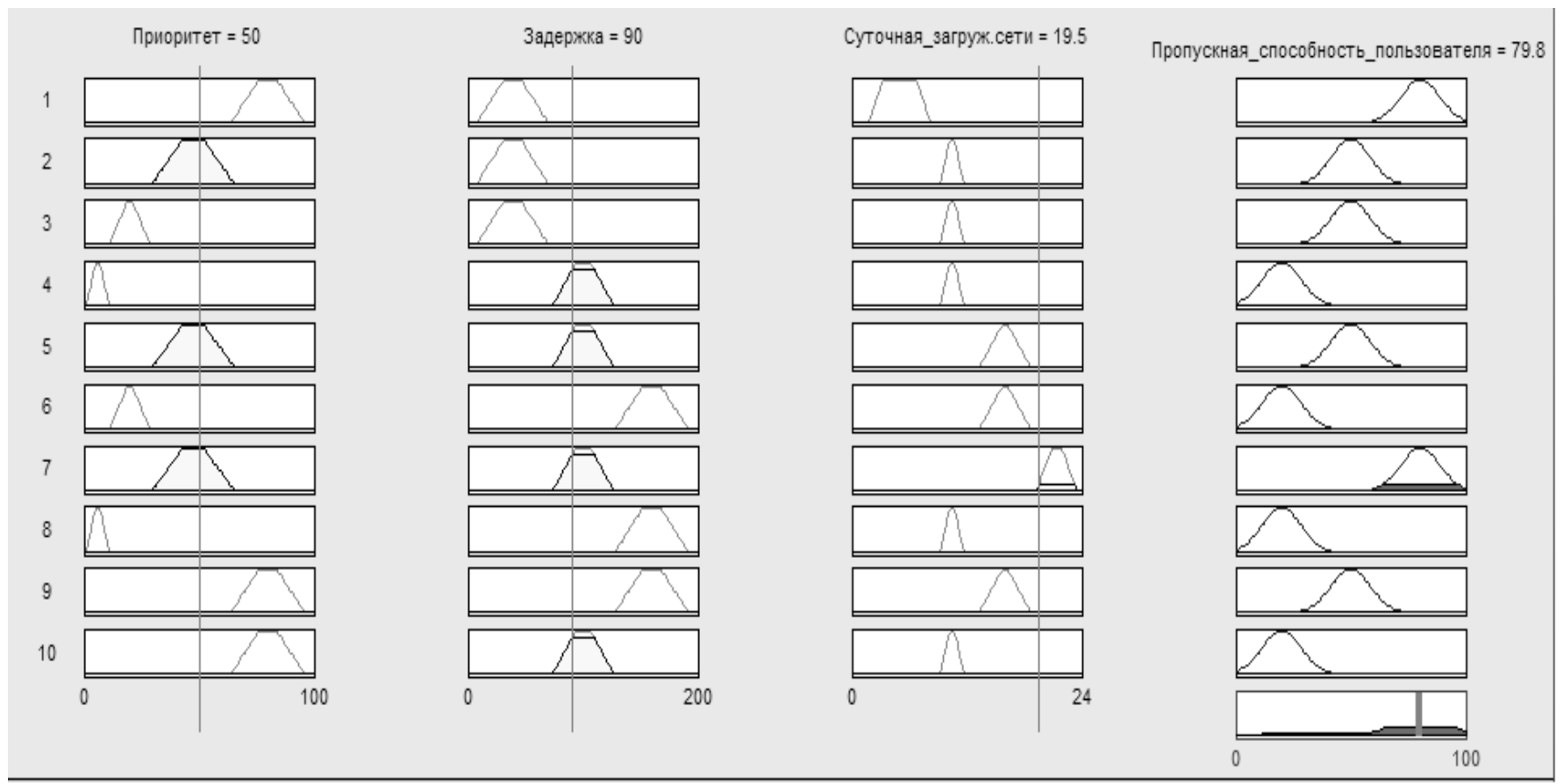

Рис. 4. Визуализация нечеткого вывода с трапециевидными функциями принадлежности

существенно упрощает необходимые аналитические и численные расчеты при применении методов теории нечетких множеств.

3. Формируется база правил нечеткого вывода для работы контроллера. Для каждой переменной определяется пропускная способность в соответствии с этой базой правил.
Формирование базы выполняется на основе экспертных знаний и включает в себя, в данном случае, 36 правил, в силу того, что каждая входная переменная принимает всего лишь по 3 или 4 различных значения.

4. На основе разработанных правил находится значение выходной лингвистической переменной (пропускной способности). В результате операции дефаззифи- 


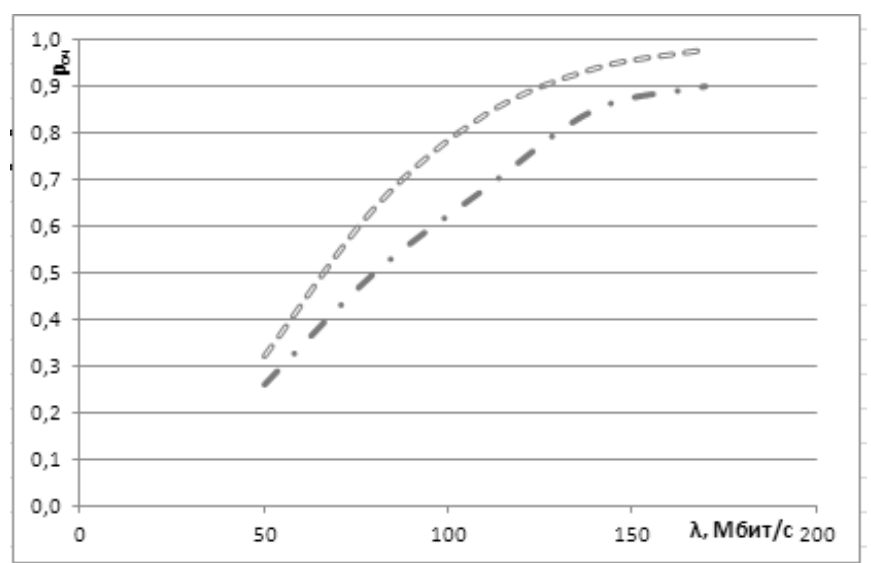

Рис. 5. График зависимости образования очереди от пропускной способности

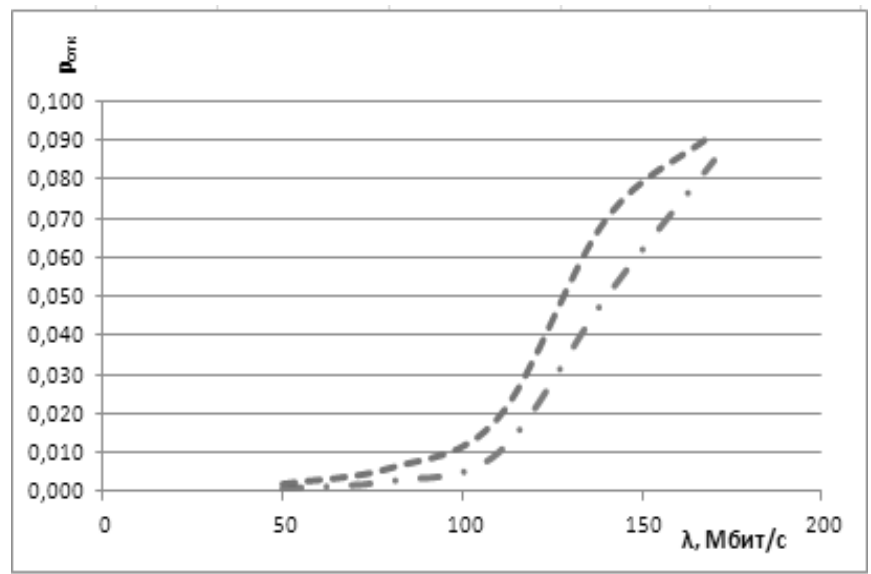

Рис. 6. График зависимости вероятности отказа от пропускной способности

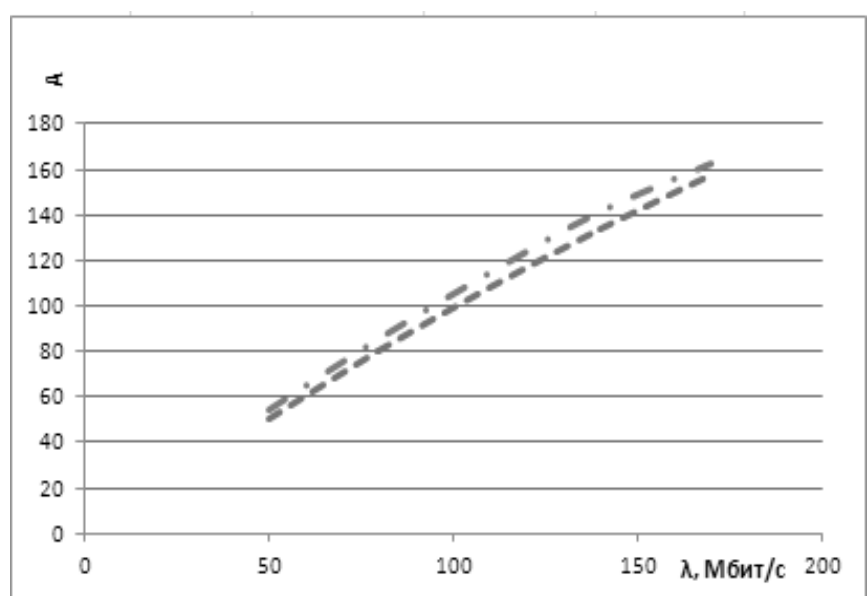

Рис. 7. График зависимости абсолютной пропускной способности от интенсивности обслуживания кации на выходе контроллера генерируется значение пропускной способности.

На рис. 2 дано визуальное представление правила для лингвистических переменных с треугольной t-нормой. Видно, что при приоритете «премиум», задержке «средняя» и загрузки сети «маленькая» пропускная способность - «высокая» (80 Мбит/с).

Выше упоминалось о типизации функций принадлежности, используемых при нечетком выводе. В частности, могут быть использованы гауссовские и трапециевидные функции принадлежности. Для рассматриваемого примера рис. 3 иллюстрирует соответствующую визуализацию нечеткого вывода при заданных значениях входных параметров. Аналогично, на рис. 4 представлена визуализация релевантного нечеткого вывода для трапециевидной функции.

Полученные результаты позволяют выявить наиболее эффективную модель функции принадлежности, которую необходимо использовать для адекватного нечеткого управления. Так в рассмотренном примере, при одинаковых входных значениях переменных: приоритет - 50\%; задержка - 90 мс; суточная загруженность сети - 19,5 ч, значения выходной переменной различны во всех трех случаях, а именно, для треугольной функции принадлежности - 80 Мбит/с; для гауссовской - 77,4 Мбит/с; для трапециевидной - 79,8 Мбит/с. Следовательно, использование треугольной функции принадлежности является предпочтительным.

\section{Обсужление полученных результатов}

Для оценки и обоснования достоверности полученных результатов рассматриваемой проблемы был использован известный аппарат теории массового обслуживания, рассчитаны основные характеристики соответствующей системы массового обслуживания (СMO) и построены графики необходимых зависимостей.

Для подтверждения достоверности полученных результатов для нового метода были построены графики зависимости основных характеристик СМО от интенсивности поступления пакетов. На рис. 5-7, соответственно, представлены графики зависимости образования очереди, вероятности отказа и абсолютной пропускной способности от пропускной способности с применением аппарата нечеткой логики и без его применения (пунктирная кривая - зависимость без применения аппарата нечеткой логики, штрихпунктирная кривая с применением аппарата).

На указанных графиках несложно заметить, что с применением аппарата нечеткой логики вероятно- 
сти отказов и образования очереди снижаются, а абсолютная пропускная способность увеличивается, что подтверждает эффективность предложенного метода и возможность его практического применения для управления информационным трафиком.

\section{Зак^ючение}

В рамках выполненного исследования рассмотрены вопросы управления информационным трафиком в телекоммуникационных сетях на основе аппарата нечеткой логики. Для основы нового алгоритма нечеткого управления выбран алгоритм Мамдани. Для реализации этих операций заданы требуемые лингвистические переменные, используемые для управления сетью, для этих переменных построены необходимые функции принадлежности трех видов. Сформирована база правил нечеткого вывода, на основе которых находится значение выходной лингвистической переменной.
На реальном примере продемонстрированы возможности применения предложенного алгоритма нечеткого управления. Полученные результаты позволили выявить наиболее эффективную модель функции принадлежности, которую необходимо использовать для адекватного нечеткого управления. Установлено, что целесообразно использовать треугольные функции принадлежности для входных и выходной переменных, поскольку их применение позволяет реализовать предоставление пользователю наибольшую пропускную способность. Для оценки и обоснования достоверности полученных результатов рассматриваемой проблемы были рассчитаны основные характеристики соответствующей системы массового обслуживания и построены графики необходимых зависимостей. Установлено, что с применением алгоритма нечеткого управления вероятности отказов и образования очереди в телекоммуникационной сети снижаются, а абсолютная пропускная способность увеличивается.

\section{ЛИТЕРАТУРА}

1. Симаков Д. В. Управление трафиком в сети с высокой динамикой метрик сетевых маршрутов//Интернет-журнал «Науковедение».2016.С.1-13

2. Bogatin E. Signal and Power Integrity Simplified/Simplified 2nd edition//Prentice. Pearson Education. 2010.p.902 — ISBN-13 978-0-13-234979-6; ISBN-10 0-13-234979-5.

3. Bouillard A., Boyer M., Le Corronc E. Deterministic Network Calculus: From Theory to Practical Implementation// New York: Wiley-ISTE. 2018. p.334.

4. Levy B. C. Random Processes with Applications to Circuits and Communications//Springer. 2019. p.466. — ISBN3030222969.

5. Moscholios I., Logothetis M. Efficient Multirate Teletraffic Loss Models Beyond Erlang//Wiley-IEEE Press.2019. p. 556 — ISBN: 978-1-119-42688-2.

6. Zukerman M. Introduction to Queueing Theory and Stochastic Teletraffic Models//ArXiv.org.2018. p.282.

7. Потапчук И. А. Метод маршрутизации в беспроводной самоорганизующейся сети на основе аппарата нечеткой логики/А.В. Багрянцев//Техника и электроника высоких напряжений.2016.С.82-88.

8. Сорокин А.А. // Адаптивное нечеткое управление заполнением буфера сетевого устройства/Научно-технические ведомости санкт-петербургского государственного политехнического университета. Информатика. Телекоммуникации. Управление.2018.С.36-48

9. Н. Паклин. Нечеткая логика-математические основы. URL: https://naukovedenie.ru/PDF/199EVN515. (дата обращения:26.10.2019).

10. Штовба С. Д. Введение в теорию нечетких множеств и нечеткую логику. URL: http://matlab.exponenta.ru/fuzzylogic .(дата 0бращения: 15.01.2020).

11. Асадулаев Р. Г. Нечеткая логика и нейронные сети//учебное пособие, Белгород, БелГУ.2017.С.309

12. Емельянов С. Г. Адаптивные нечетко-логические системы управления/В.С. Титов, М. В. Бобырь//Москва. 2013.С.193

13. Бауэр П. Введение в нечеткую логику и системы нечеткого управления. URL: http://www.gotai.net/documents/doc-I-fl-001.aspx. (дата обращения:22.02.2020).

(c) Матрохина Кристина Васильевна (k.matrokhina@mail.ru).

Журнал «Современная наука: актуальные проблемы теории и практики» 